\title{
Coupling between hyperbolic and diffusive systems: a port-Hamiltonian formulation $\dagger$
}

\author{
Yann Le Gorrec ${ }^{1, *}$ and Denis Matignon ${ }^{2, *}$
}

\begin{abstract}
The aim of this paper is to study a conservative wave equation coupled to a diffusion equation. This coupled system naturally arises in musical acoustics when viscous and thermal effects at the wall of the duct of a wind instrument are taken into account. The resulting equation, known as Webster-Lokshin model, has variable coefficients in space, and a fractional derivative in time. This equation can be recast into the port Hamiltonian framework by using the diffusive representation of the fractional derivative in time and a multiscale state space representation. The port-Hamiltonian formalism proves adequate to reformulate this coupled system, and could enable another well-posedness analysis, using classical results from port-Hamiltonian systems theory.
\end{abstract}

Keywords: Energy storage, port-Hamiltonian systems, partial differential equations, fractional derivatives, diffusive representation.

\section{INTRODUCTION}

The dissipative model which describes acoustic waves traveling in a duct with viscothermal losses at the lateral walls is a wave equation with spatially-varying coefficients, which involves fractional-order integrals and derivatives with respect to time [Polak(1991)]. This model is first rewritten in a coupled form; then the fractional integrals and derivatives are written in their so-called diffusive representation; essentially, the fractional-order time kernel in the integral is represented by its Laplace transform.

The main idea of the present work is to put the Webster-Lokshin fractional PDE into the infinite dimensional port-Hamiltonian framework [van der Schaft et al.(2002)], [Le Gorrec et al.(2005)], in order to take advantage of this setting. Port Hamiltonian framework has shown to be very powerful to prove the existence and the well posedness of solutions and to prove the stability of infinite dimensional systems [Zwart et al.(2010)], [Villegas et al.(2009)]. To do so, a preliminary work is necessary, that is using diffusive representations of both fractional integrals and derivatives in order to imagine the ad hoc Hamiltonian formulation as a multiscale coupling of two port Hamiltonian systems. This

$\dagger$ A first version of this work has been presented at the IFAC conference on Lagrangian and Hamiltonian Methods for Nonlinear Control, LMNHC 2012, see [Le Gorrec et al.(2012)].

* The contribution of both authors has been done within the context of the French National Research Agency sponsored project HAMECMOPSYS. Further information is available at http: / / www . hamecmopsys.ens $2 \mathrm{~m}$. fr/.

${ }^{1} \mathrm{Y}$. Le Gorrec is with the FEMTO-ST institute, ENSMM 24 rue Alain Savary, 25000 Besançon, France. Yann.Le.Gorrec at ens $2 \mathrm{~m}$. fr

${ }^{2} \mathrm{D}$. Matignon is with the University of Toulouse, ISAE; 10, av. E. Belin; BP 54032. 31055 Toulouse Cedex 4, France denis.matignon at isae.fr work has to be related to the multiscale coupling proposed in [Baaiu et al.(2009)]. The coupling between conservative and dissipative subsystems is then easily tackled in this setting; but for the PDE, as usual, some care must be taken with the functional setting.

The outline of the paper is as follows: section $\S$ II starts with some background on port-Hamiltonian systems, both in finite dimension and infinite dimension, with an emphasis on the modelling of damping, see II-A; some specific damping models are then examined in $\S$ II-B: fractional integrals and derivatives are defined first, and diffusive representations are introduced in order to replace these fractional operators by input-output representations, and state-space representation, which prove compatible with first order dynamical systems. In order to set up a Hamiltonian formulation of both these operators, a finite-dimensional toy-model is studied in depth in $\S$ III: ad hoc discrete energies are being defined, skewsymmetric and symmetric structural matrices $J$ and $R$ are identified, and the standard port-Hamiltonian structure of dissipative systems is recovered. Finally, the fully infinitedimensional case is presented in $\S$ IV: the Webster-Lokshin model is recast in the setting of infinite-dimensional portHamiltonian systems with dissipation. The paper ends in $\S \mathrm{V}$ with many perspectives of this ongoing work.

\section{SOME BACKGROUND ON PORT HAMILTONIAN SYSTEMS AND DIFFUSIVE SYSTEMS}

\section{A. Port Hamiltonian systems}

1) The finite dimensional case: Port Hamiltonian systems (PHS) [van der Schaft and Maschke(2004)] have been widely used in modelling and control of mechanical and electromechanical systems, see also [Duindam et al.(2009)]. It has first been defined from Dirac structures (arising from the use of power conjugate variables and the skew symmetry of the interconnection structure) in the case of power preserving systems. In the case of systems with dissipation, PHS are defined by:

$$
\left\{\begin{array}{l}
\dot{X}=(J(X)-R(X)) \partial_{X} \mathcal{H}(X)+g(X) u(t) \\
y=g(X)^{T} \partial_{X} \mathcal{H}(X)
\end{array}\right.
$$

where $X \in \mathbb{R}^{n}, \mathcal{H}(X)$ is the Hamiltonian function usually chosen as the total energy of the system, $\partial_{X} \mathcal{H}(X)$ is the vector of driving forces, $J(X)=-J(X)^{T}$ and $R(X)=$ $R(X)^{T} \geq 0$ which may depend on the state vector $X$ specify the interconnection matrix and the dissipation matrix of the system respectively, and $g(X)$ is the matrix of smooth input mapping functions. The energy balance associated to this 
system is:

$\frac{d \mathcal{H}}{d t}=\left(\partial_{X} \mathcal{H}\right)^{T} \frac{\partial X}{\partial t}=y^{T} u(t)-\underbrace{\left(\partial_{X} \mathcal{H}(X)\right)^{T} R\left(\partial_{X} \mathcal{H}(X)\right)}_{d \geq 0}$

In the case of linear systems the energy can be written as a quadratic form $\mathcal{H}(X)=\frac{1}{2} X^{T} L X$ where $L$ is symmetric positive definite and is related to the physical parameters of the system.

Example 1 (Mass spring system). We consider the simple mass spring system example. A spring on a support (we do not consider the gravity force) is fixed at one end and attached to a load at the other end. A force $F(t)$ is applied to the load. From Newton's second law:

$$
m \ddot{x}=-\kappa x+F
$$

where $x(t)$ is the relative position of the load, $m$ the mass of the load, $F$ the applied force, and $\kappa$ the stiffness of the spring. Let's now consider the position $q=x$ and the momentum $p=m \dot{x}$ (i.e. the energy variables) as state variables $X(t)=$ $(q(t) p(t))^{T}$. The energy of the system is given by the sum of the elastic potential energy and the kinetic energy:

$\mathcal{H}(q, p)=\frac{1}{2}\left(\kappa q^{2}+\frac{p^{2}}{m}\right)=\frac{1}{2}\left(\begin{array}{ll}q & p\end{array}\right) \underbrace{\left(\begin{array}{cc}\kappa & 0 \\ 0 & \frac{1}{m}\end{array}\right)}_{L>0}\left(\begin{array}{c}q \\ p\end{array}\right)$

System (1) can be written as a port-Hamiltonian system:

$$
\begin{aligned}
& \left(\begin{array}{c}
\dot{q} \\
\dot{p}
\end{array}\right)=\underbrace{\left(\begin{array}{cc}
0 & 1 \\
-1 & 0
\end{array}\right)}_{J} \underbrace{\left(\begin{array}{cc}
\kappa & 0 \\
0 & \frac{1}{m}
\end{array}\right)\left(\begin{array}{l}
q \\
p
\end{array}\right)}_{\partial_{X} \mathcal{H}(X)=L X}+\underbrace{\left(\begin{array}{l}
0 \\
1
\end{array}\right)}_{g(x)} F \\
& y=\left(\begin{array}{ll}
0 & 1
\end{array}\right)\left(\begin{array}{cc}
\kappa & 0 \\
0 & \frac{1}{m}
\end{array}\right)\left(\begin{array}{c}
q \\
p
\end{array}\right)=\dot{x}
\end{aligned}
$$

In this case the energy is preserved and the skew symmetry of the interconnection structure implies that the internal variation of the energy comes from the power exchanged with the environment. It is a direct consequence of the energy balance:

$$
\frac{d \mathcal{H}(X)}{d t}=\frac{\partial \mathcal{H}(X)^{T}}{\partial X} \frac{d X}{d t}=F \dot{x}
$$

When viscous damping with constant coefficient $\varepsilon$ is considered, equation (1) becomes:

$$
m \ddot{x}=-\kappa x-\varepsilon \dot{x}+F
$$

leading to:

$$
\begin{aligned}
& \left(\begin{array}{l}
q \\
p
\end{array}\right)=\underbrace{\left(\begin{array}{cc}
0 & 1 \\
-1 & -\varepsilon
\end{array}\right)}_{J-R} \underbrace{\left(\begin{array}{cc}
\kappa & 0 \\
0 & \frac{1}{m}
\end{array}\right)\left(\begin{array}{l}
q \\
p
\end{array}\right)}_{\partial_{X} \mathcal{H}(X)=L X}+\underbrace{\left(\begin{array}{l}
0 \\
1
\end{array}\right)}_{g(X)} F \\
& y=\left(\begin{array}{ll}
0 & 1
\end{array}\right)\left(\begin{array}{cc}
\kappa & 0 \\
0 & \frac{1}{m}
\end{array}\right)\left(\begin{array}{l}
q \\
p
\end{array}\right)=\dot{x}
\end{aligned}
$$

We can note that the dissipation is expressed through the positivity of the $R$ term, leading to the balance equation:

$$
\frac{d \mathcal{H}(X)}{d t}=F \dot{x}-\varepsilon \dot{x}^{2}
$$

2) Infinite dimensional port-Hamiltonian systems: Port Hamiltonian systems have been extended to the case of distributed parameter systems and more specifically in the case of power conservative linear systems defined on one dimensional spatial domain $(z \in[a, b])$ by using real Hilbert spaces in [Le Gorrec et al.(2005)] and in the case of system with dissipation in [Villegas et al.(2006)], [Le Gorrec et al.(2006)]. In this later case the associate PDE is of the form:

$$
\dot{X}(z, t)=(\mathcal{J}(X)-\mathcal{R}(X)) \mathcal{L}_{z} X(z, t),
$$

with $\mathcal{J}(X)$ a formally skew symmetric differential operator of order $N$ and $X(z, t) \in H^{N}\left((a, b) ; \mathbb{R}^{n}\right), \mathcal{R}=\mathcal{G S G}^{*}$ a formally skew symmetric differential operator of degree less or equal to $N, S$ and $\mathcal{L}_{z}$ two coercive operators that may depend on $z$. The total energy of the system is defined as

$$
\mathcal{H}(X)=\frac{1}{2} X(z, t)^{T} \mathcal{L}_{z} X(z, t)
$$

The definition of infinite dimensional port-Hamiltonian systems is fundamentally linked to the definition of the port variables, derived in the power conservative case $(\mathcal{R}(X)=$ $0)$ from the skew symmetry of the operator in the case of open systems, and from which the Dirac structure is defined. In the case of systems of the form (5) i.e. with dissipation, the overall differential operator is not skew symmetric. Yet, a Dirac structure can be associated with the interconnection structure (that is skew symmetric) defined by the extended skew symmetric operator $\mathcal{J}_{e}$ as follows:

$$
\left(\begin{array}{c}
\dot{X}(z, t) \\
f_{s}
\end{array}\right)=\underbrace{\left(\begin{array}{cc}
\mathcal{J} & \mathcal{G} \\
-\mathcal{G}^{*} & 0
\end{array}\right)}_{\mathcal{J}_{e}}\left(\begin{array}{c}
\mathcal{L} X(z, t) \\
e_{s}
\end{array}\right)
$$

and the closure relation:

$$
e_{s}=S f_{s}
$$

which is equivalent to (5). From a geometrical point of view (5) can be written:

$$
\left(\begin{array}{c}
f \\
f_{s}
\end{array}\right)=\mathcal{J}_{e}\left(\begin{array}{c}
e \\
e_{s}
\end{array}\right) \Leftrightarrow f_{e}=\mathcal{J}_{e} e_{e}
$$

More precisely the Dirac structure can be defined as follows.

Definition 1. Let consider that $e_{e} \in \mathcal{E}_{e}$ and $f_{e} \in \mathcal{F}_{e}$ with values at the boundary $e_{\partial} \in \mathcal{E}_{\partial}=\mathbb{R}^{n N}$ and $f_{\partial} \in \mathcal{F}_{\partial}=$ $\mathbb{R}^{n N}$ where $\mathcal{E}_{e}$ and $\mathcal{F}_{e}$ are real Hilbert spaces. A Dirac structure $\mathcal{D}$ on the bond space $\mathcal{B}=\mathcal{E} \times \mathcal{E}_{\partial} \times \mathcal{F} \times \mathcal{F}_{\partial}$ is a subspace of $\mathcal{B}$ which is maximally isotropic with respect to the canonical symmetrical pairing (with $b, \tilde{b} \in \mathcal{B}$ )

$$
\begin{aligned}
\langle b, \tilde{b}\rangle_{+} & =\left\langle\left(e_{e}, \tilde{f}_{e}\right)\right\rangle+\left\langle\left(\tilde{e}_{e}, f_{e}\right)\right\rangle \\
& -\left\langle\left(\tilde{e}_{\partial}, f_{\partial}\right)\right\rangle-\left\langle\left(e_{\partial}, \tilde{f}_{\partial}\right)\right\rangle
\end{aligned}
$$

i.e.,

$$
\mathcal{D}^{\perp}=\mathcal{D}
$$

A constructive definition of the boundary port variables in the case of operators of the form $(N=1)$ :

$$
\mathcal{J}=P_{1} \frac{\partial}{\partial z}+P_{0}, \quad \text { and } \quad \mathcal{G}=G_{1} \frac{\partial}{\partial z}+G_{0}, P_{i}, G_{i} \in \mathbb{R}^{n}
$$


with $P_{0}=-P_{0}^{T}, P_{1}=P_{1}^{T}$ can be found in [Villegas et al.(2006)], [Jacob et al.(2012)] and is recalled in Definition 2.

Definition 2. Let $r$ be the rank of $\tilde{P}_{1}=\left(\begin{array}{cc}P_{1} & G_{1} \\ -G_{1}^{T} & 0\end{array}\right)$ and $M$ be an $n \times r$ matrix, whose columns are linearly independent and span the range of $\tilde{P}_{1}$. Let define: $Q=$ $M^{T} P_{1} M$ and $M_{Q}=\left(M^{T} M\right)^{-1} M^{T}$ (Note that if $P_{1}$ is full rank $M=I$ ).

The boundary port variables associated with the differential operator $\mathcal{J}_{e}$ are the vectors $e_{\partial}, f_{\partial} \in \mathbb{R}^{n}$, defined by

$$
\left(\begin{array}{c}
f_{\partial} \\
e_{\partial}
\end{array}\right)=R_{\mathrm{ext}}\left(\begin{array}{c}
M_{Q} e_{e}(b) \\
M_{Q} e_{e}(a)
\end{array}\right)
$$

where $R_{\text {ext }}$ is defined by:

$$
R_{\mathrm{ext}}=\frac{1}{\sqrt{2}}\left(\begin{array}{cc}
Q & -Q \\
I & I
\end{array}\right)
$$

Remark 1. In one space dimension, functions belonging to $H^{1}\left((a, b) ; \mathbb{R}^{n}\right)$ are necessarily continuous, thus using pointwise values at the boundary, such as $e_{e}(a)$ and $e_{e}(b)$ in (8) definitely makes sense.

In higher space dimensions though, the trace operator must be used to define variables at boundary of the spatial domain.

Then, it is possible to derive the Dirac structure associated with (7).

Theorem 1. [Villegas et al.(2006)]Let $H^{1}\left((a, b) ; \mathbb{R}^{n}\right)$ denote the Sobolev space of weakly differentiable functions on the interval $(a, b)$. The subspace $\mathcal{D}_{\mathcal{J}_{e}}$ defined as

$$
\begin{aligned}
& \mathcal{D}_{\mathcal{J}_{e}}=\left\{\left(\begin{array}{c}
f_{e} \\
f_{\partial} \\
e_{e} \\
e_{\partial}
\end{array}\right) \mid e_{e} \in H^{1}\left((a, b) ; \mathbb{R}^{n}\right) \times H^{1}\left((a, b) ; \mathbb{R}^{n}\right),\right. \\
& \left.f_{e}=\mathcal{J}_{e} e_{e},\left(\begin{array}{l}
f_{\partial} \\
e_{\partial}
\end{array}\right)=R_{\mathrm{ext}}\left(\begin{array}{c}
M_{Q} e_{e}(b) \\
M_{Q} e_{e}(a)
\end{array}\right)\right\}
\end{aligned}
$$

\section{is a Dirac structure.}

The definition of a port-Hamiltonian system derives directly from the definition of Dirac structure.

Definition 3. An infinite-dimensional port-Hamiltonian system with dissipation is defined by:

$$
\left(\dot{x}, f_{s}, f_{\partial}, \delta_{x} \mathcal{H}(x), e_{s}, e_{\partial}\right) \in \mathcal{D}_{\mathcal{J}_{e}}
$$

where $e_{s}=S f_{s}$ with $S>0$ and $\delta_{x} \mathcal{H}(x)$ the variational derivative of $\mathcal{H}(x)$.

Remark 2. If $P_{1}$ is full rank and $G_{1}=0$ then

$$
M=\left(\begin{array}{c}
I_{n} \\
0
\end{array}\right) \text { and } M_{Q}=\left(\begin{array}{ll}
I_{n} & 0
\end{array}\right)
$$

\section{B. Diffusive systems}

Some damping models involve fractional derivatives with respect to the time variable: these causal linear operators can be defined in many ways, and we first give a recap of the definitions in the time domain, first of fractional integrals of order $\beta$, then of fractional derivatives of order $\alpha$, see e.g. [Matignon(2009a)].

1) Fractional integrals and derivatives: Let $\beta \in(0,1)$, and $\operatorname{set}^{1} h_{\beta}(t):=\frac{1}{\Gamma(\beta)} t^{\beta-1}$ for $t>0$ only; then, $h_{\beta} \in$ $L_{\text {loc }}^{1}\left(\mathbb{R}^{+}\right)$. For any $T>0$, let $u \in L^{2}(0, T)$, and define $I^{\beta} u:=h_{\beta} \star u$ or, more explicitely:

$$
I^{\beta} u(t)=\int_{0}^{t} \frac{1}{\Gamma(\beta)} \tau^{\beta-1} u(t-\tau) \mathrm{d} \tau .
$$

This is the Riemann-Liouville fractional integral of order $\beta \in$ $(0,1)$ of $u$ : it is causal, and belongs to $L^{2}(0, T)$ also. In terms of causal Laplace transform, $H_{\beta}(s)=s^{-\beta}$ in $\Re e(s)>0$; hence, the interpretation of the fractional integral is a causal low-pass filter, with a gain of $-6 \beta \mathrm{d} B$ per octave.

The fractional derivative is the inverse of the fractional integral, but some technicalities are to be found in this case. Let $\alpha \in(0,1)$, and for any $T>0$, let $u \in H^{1}(0, T)$, (that is $u \in L^{2}(0, T), u$ has a weak derivative say $\dot{u}$ which does belong to $L^{2}(0, T)$ ), and define $D^{\alpha} u=I^{1-\alpha} D u:=h_{1-\alpha} \star \dot{u}$ or, more explicitely:

$$
D^{\alpha} u(t)=\int_{0}^{t} \frac{1}{\Gamma(1-\alpha)} \tau^{-\alpha} \dot{u}(t-\tau) \mathrm{d} \tau .
$$

This is the fractional derivative of order $\alpha \in(0,1)$ of $u$ : it is causal, and belongs to $L^{2}(0, T)$. In terms of causal Laplace transform, $\widetilde{H}_{\alpha}(s)=s^{+\alpha}$ in $\Re e(s)>0$; hence, the interpretation of the fractional derivative is a causal high-pass filter, with a gain of $+6 \alpha \mathrm{d} B$ per octave.

In the next sections, we shall make a link between these fractional operators, and ordinary differential equations (ODEs), or just convolution by families of decaying exponentials: beginning by an easy numerical identity, we are able to derive functional identities for the kernel $h_{\beta}$ and its Laplace transform $H_{\beta}$, in the time-domain and the frequency-domain respectively; these are being used to define input-output representations, and even state-space realizations which will be of great help in the sequel; part of this presentation is borrowed from [Matignon(2009b)].

2) An Elementary Approach: Consider the numerical identity, valid for $\delta>1$ :

$$
\int_{0}^{\infty} \frac{\mathrm{d} x}{1+x^{\delta}}=\frac{\frac{\pi}{\delta}}{\sin \left(\frac{\pi}{\delta}\right)}
$$

Letting $s \in \mathbb{R}_{*}^{+}$and substituting $x=\left(\frac{\xi}{s}\right)^{\frac{1}{\delta}}$ in the above numerical identity, we get:

$$
\int_{0}^{\infty} \frac{\sin \left(\frac{\pi}{\delta}\right)}{\pi} \frac{1}{\xi^{1-\frac{1}{\delta}}} \frac{1}{s+\xi} d \xi=\frac{1}{s^{1-\frac{1}{\delta}}}
$$

Finally, performing an analytic continuation from $\mathbb{R}^{+*}$ to $\mathbb{C} \backslash \mathbb{R}^{-}$for both sides of the above identity in the complex

\footnotetext{
${ }^{1} \Gamma(\beta)$ is a function of $\beta$
} 
variable $s$, and letting $\beta:=1-\frac{1}{\delta} \in(0,1)$, we get the functional identity:

$$
\begin{aligned}
H_{\beta}: \mathbb{C} \backslash \mathbb{R}^{-} & \rightarrow \mathbb{C} \\
s & \mapsto \int_{0}^{\infty} \mu_{\beta}(\xi) \frac{1}{s+\xi} \mathrm{d} \xi=\frac{1}{s^{\beta}},
\end{aligned}
$$

with density $\mu_{\beta}(\xi)=\frac{\sin (\beta \pi)}{\pi} \xi^{-\beta}$.

Applying an inverse Laplace transform to both sides gives:

$$
\begin{aligned}
h_{\beta}: \mathbb{R}^{+} & \rightarrow \mathbb{R} \\
t & \mapsto \int_{0}^{\infty} \mu_{\beta}(\xi) e^{-\xi t} \mathrm{~d} \xi=\frac{1}{\Gamma(\beta)} t^{\beta-1} .
\end{aligned}
$$

3) Input-output Representations: Let $u$ and $y:=I^{\beta} u$ be the input and output of the causal fractional integral of order $\beta$. Using the integral representations above, together with Fubini's theorem, we get:

$$
y(t)=\int_{0}^{\infty} \mu_{\beta}(\xi)\left[e_{\xi} \star u\right](t) \mathrm{d} \xi,
$$

with $e_{\xi}(t):=e^{-\xi t}$, and $\left[e_{\xi} \star u\right](t)=\int_{0}^{t} e^{-\xi(t-\tau)} u(\tau) \mathrm{d} \tau$.

Now for fractional derivative of order $\alpha \in(0,1)$, we have $\widetilde{y}=D^{\alpha} u=D\left[I^{1-\alpha} u\right]$, and a careful computation shows that:

$$
\widetilde{y}(t)=\int_{0}^{\infty} \mu_{1-\alpha}(\xi)\left[u-\xi e_{\xi} \star u\right](t) \mathrm{d} \xi .
$$

4) State Space Representation: In both input-output representations above, introducing a state, say $\varphi(\xi,$.$) which$ realizes the classical convolution $\varphi(\xi,):.=\left[e_{\xi} \star u\right](t)$ leads to the following diffusive realizations, in the sense of systems theory:

$$
\begin{aligned}
\partial_{t} \varphi(\xi, t) & =-\xi \varphi(\xi, t)+u(t), \varphi(\xi, 0)=0, \\
y(t) & =\int_{0}^{\infty} \mu_{\beta}(\xi) \varphi(\xi, t) \mathrm{d} \xi
\end{aligned}
$$

and

$$
\begin{aligned}
\partial_{t} \widetilde{\varphi}(\xi, t) & =-\xi \widetilde{\varphi}(\xi, t)+u(t), \widetilde{\varphi}(\xi, 0)=0 \\
\widetilde{y}(t) & =\int_{0}^{\infty} \mu_{1-\alpha}(\xi)[u(t)-\xi \widetilde{\varphi}(\xi, t)] \mathrm{d} \xi
\end{aligned}
$$

These are first and extended diffusive realizations, respectively. The slight difference between (13)-(14) and (15)-(16), marked by the $\sim$ notation, lies in the underlying functional spaces in which these equations make sense: $\varphi$ belongs to $\mathcal{H}_{\beta}:=\left\{\varphi\right.$ s.t. $\left.\int_{0}^{\infty} \mu_{\beta}(\xi)|\varphi|^{2} \mathrm{~d} \xi<\infty\right\}$, whereas $\widetilde{\varphi}$ belongs to $\widetilde{\mathcal{H}}_{\alpha}:=\left\{\widetilde{\varphi}\right.$ s.t. $\left.\int_{0}^{\infty} \mu_{1-\alpha}(\xi)|\widetilde{\varphi}|^{2} \xi \mathrm{d} \xi<\infty\right\}$, see e.g. [Haddar et al.(2008), ch. 2], or [Matignon et al.(2013b].

\section{A TOY MODEL.}

In this section, we first consider a classical mechanical oscillator with fluid damping in $\S$ III-A, then we use the velocity as input of two different types of damping models: a low-pass diffusive subsystem (such as a discretized fractional integral) in $\S$ III-B, or a high-pass diffusive subsystem (such as a discretized fractional derivative) in $\S$ III-C.

\section{A. Mass spring system with damping}

We start with the port-Hamiltonian formulation of the single finite dimensional harmonic oscillator. Dynamic equation is usually written in the form:

$$
m \ddot{x}+\varepsilon \dot{x}+\kappa x=0
$$

where $x(t) \in \mathbb{R}$ and $m, \varepsilon, \kappa$ are positive constants. By using as state variables the energy variables (1.e. the position and the momentum) and defining the Hamiltonian $\mathcal{H}_{0}$ as the total energy of the system, i.e.:

$$
X:=\left[\begin{array}{c}
q=x, \\
p=m \dot{x}
\end{array}\right] \quad \text { and } \quad \mathcal{H}_{0}(X)=\frac{1}{2 m} p^{2}+\frac{1}{2} \kappa x^{2} ;
$$

it is possible to rewrite (17) in the form of a port-Hamiltonian system:

$$
\begin{aligned}
& \qquad \frac{d}{d t} X=\left[\begin{array}{cc}
0 & 1 \\
-1 & -\varepsilon
\end{array}\right] \partial_{X} \mathcal{H}_{0}(X)=\left(J-R_{\varepsilon}\right) \partial_{X} \mathcal{H}_{0}(X) \\
& \text { where } \partial_{X} \mathcal{H}_{0}(X)=\left[\begin{array}{c}
\kappa x=\kappa x \\
\frac{1}{m} p=\dot{x}=v
\end{array}\right] \text { and: } \\
& \qquad J=\left[\begin{array}{cc}
0 & 1 \\
-1 & 0
\end{array}\right] \quad \text { and } \quad R_{\varepsilon}=\left[\begin{array}{ll}
0 & 0 \\
0 & \varepsilon
\end{array}\right]
\end{aligned}
$$

$J$ is full rank $n=2$ and skew-symmetric, whereas $R_{\varepsilon}$ is symmetric positive $(\varepsilon>0)$, with rank equal to 1 , thus not positive definite.

\section{B. Coupling with a low-pass diffusive system}

The damping model is now given by the coupling with another dynamical system, the input of which is the velocity $v:=\dot{x}$, and the output of which is $y$, a positive linear combination of first-order low-pass subsytems, as follows:

$$
\begin{aligned}
m \ddot{x}+y+\kappa x & =0, \quad \text { with } \quad y=\sum_{k=1}^{K} \mu_{k} \varphi_{k} \\
\text { where } \dot{\varphi}_{k} & =-\xi_{k} \varphi_{k}+v, \quad \text { for } 1 \leq k \leq K .
\end{aligned}
$$

Hence, with $\mathcal{H}_{\Phi}:=\frac{1}{2} \sum_{k=1}^{K} \mu_{k} \varphi_{k}^{2}$, and $\partial_{\varphi_{k}} \mathcal{H}_{\Phi}=\mu_{k} \varphi_{k}$, the total system can be described by an extended state $X=$ $(x, p, \Phi)$ and a total Hamiltonian $\mathcal{H}:=\mathcal{H}_{0}+\mathcal{H}_{\Phi}$.

$$
\frac{d}{d t} X=\left[\begin{array}{ccc}
0 & 1 & 0 \\
-1 & 0 & -\mathbb{1}^{T} \\
0 & \mathbb{1} & -\operatorname{diag}\left(\frac{\xi_{k}}{\mu_{k}}\right)
\end{array}\right] \partial_{X} \mathcal{H}(X)=(J-R) \partial_{X} \mathcal{H}(X)
$$

In this case, matrices of size $(2+K) \times(2+K)$ are given by:

$$
J=\left[\begin{array}{ccc}
0 & 1 & 0 \\
-1 & 0 & -\mathbb{1}^{T} \\
0 & \mathbb{1} & 0
\end{array}\right] \quad \text { and } \quad R=\left[\begin{array}{ccc}
0 & 0 & 0 \\
0 & 0 & 0 \\
0 & 0 & \operatorname{diag}\left(\frac{\xi_{k}}{\mu_{k}}\right)
\end{array}\right]
$$

It can easily be checked that $J$ is skew-symmetric with rank 2 only, and $R$ is symmetric positive $\left(\xi_{k}>0, \mu_{k}>0\right)$, but not positive definite (its rank is $K$ ); its structure is simply diagonal. 
Remark 3. Note that the relation between $v$ and $y$, with transfer function $H_{K}(s)=\sum_{k=1}^{K} \mu_{k} \frac{1}{s+\xi_{k}}$, comes from a possible discretization of a diffusive system, the general structure of which would be given by the following transfer function, namely:

$$
G(s)=\int_{0}^{\infty} \mu_{\beta}(\xi) \frac{1}{s+\xi} \mathrm{d} \xi
$$

As particular and noteworthy case, if $\mu_{\beta}(\xi)=\frac{\sin (\beta \pi)}{\pi} \xi^{-\beta}$, then $G_{\beta}(s)=\frac{1}{s^{\beta}}$ is recovered, which is nothing but the fractional integral of order $\beta \in(0,1)$, a low-pass filter.

\section{Coupling with a high-pass diffusive system}

The damping model is now given by the coupling with another dynamical system, the input of which is the velocity $v:=\dot{x}$, and the output of which is $\widetilde{y}$, a positive linear combination of first-order high-pass subsytems, with a feedthrough term, $d:=\sum_{l=1}^{L} \nu_{l}$, as follows:

$$
\begin{aligned}
m \ddot{x}+\widetilde{y}+\kappa x & =0, \quad \text { with } \quad \widetilde{y}=\sum_{l=1}^{L} \nu_{l} \dot{\widetilde{\varphi}}_{l} \\
\text { where } \dot{\tilde{\varphi}}_{l} & =-\xi_{l} \widetilde{\varphi}_{l}+v, \quad \text { for } 1 \leq l \leq L .
\end{aligned}
$$

Hence, with $\mathcal{H}_{\widetilde{\Phi}}:=\frac{1}{2} \sum_{l=1}^{L} \nu_{l} \xi_{l} \widetilde{\varphi}_{l}^{2}$, and $\partial_{\widetilde{\varphi}_{l}} \mathcal{H}_{\widetilde{\Phi}}=$ $\nu_{l} \xi_{l} \widetilde{\varphi}_{l}$, the total system can be described by an extended state $X=(x, p, \widetilde{\Phi})$ and a total Hamiltonian $\mathcal{H}:=\mathcal{H}_{0}+\mathcal{H}_{\widetilde{\Phi}}$.

$\frac{d}{d t} X=\left[\begin{array}{ccc}0 & 1 & 0 \\ -1 & -d & \mathbb{1}^{T} \\ 0 & \mathbb{1} & -\operatorname{diag}\left(\frac{1}{\nu_{l}}\right)\end{array}\right] \partial_{X} \mathcal{H}(X)=(J-R) \partial_{X} \mathcal{H}(X)$ In this case, matrices of size $(2+L) \times(2+L)$ are given by:

$$
J=\left[\begin{array}{ccc}
0 & 1 & 0 \\
-1 & 0 & 0 \\
0 & 0 & 0
\end{array}\right] \quad \text { et } \quad R=\left[\begin{array}{ccc}
0 & 0 & 0 \\
0 & \sum_{l=1}^{L} \nu_{l} & -\mathbb{1}^{T} \\
0 & -\mathbb{1} & \operatorname{diag}\left(\frac{1}{\nu_{l}}\right)
\end{array}\right]
$$

It can easily be checked that $J$ is skew-symmetric with rank 2 only, and $R$ is symmetric positive $\left(\xi_{l}>0, \nu_{l}>0\right)$, but not positive definite (its rank is at most $L$ ); its structure is not that simple, but a block computation shows that $X^{T} R X=$ $\sum_{l=1}^{L}\left(\sqrt{\nu_{l}} p-\frac{1}{\sqrt{\nu_{l}}} \widetilde{\varphi}_{l}\right)^{2} \geq 0$.

Remark 4. Note that the relation between $v$ and $\widetilde{y}$, with transfer function $\widetilde{G_{L}}(s)=\sum_{k=1}^{L} \nu_{l} \frac{s}{s+\xi_{l}}$, comes from a possible discretization of a diffusive system, the general structure of which would be given by the following transfer function, namely:

$$
\widetilde{G}(s)=\int_{0}^{\infty} \nu(\xi) \frac{s}{s+\xi} \mathrm{d} \xi .
$$

As particular and noteworthy case, if $\nu_{\alpha}(\xi)=\frac{\sin (\alpha \pi)}{\pi} \xi^{\alpha-1}$, then $G_{\alpha}(s)=s^{\alpha}$ is recovered, which is nothing but the fractional derivative of order $\alpha \in(0,1)$, a high-pass filter.

\section{A HAMILTONIAN FORMULATION FOR WEBSTER-LOKSHIN MODEL}

Let now consider the Webster-Lokshin (cf. [Polak(1991)], [Hélie et al.(2006)]) equation in PHS format. It is given in the usual PDE form²

$$
\partial_{t}^{2} w+\left(\varepsilon_{z} \partial_{t}^{1 / 2}+\eta_{z} \partial_{t}^{-1 / 2}\right) \partial_{t} w-\frac{1}{r_{z}^{2}} \partial_{z}\left(r_{z}^{2} \partial_{z} w\right)=0 \text {. }
$$

where

$$
w=w(t, z)
$$

Here, coefficient $\varepsilon_{z}>0$ is conversely proportional to the radius $r_{z}$, and the proportionality constants involved are linked to the square roots of $l_{v}$ and $l_{h}$, that are the characteristic lengths of viscous and thermal effects, respectively [Polak(1991)]. Also coefficient $\eta_{z}>0$ appears in higher order developpements, in an asymptotic analysis procedure [Kergomard et al.(2013)].

Using the diffusive representation of $\S$ II-B, Equation (18) can be written:

$$
\partial_{t}^{2} w+\left(\varepsilon_{z} \widetilde{y}+\eta_{z} y\right)-\frac{1}{r_{z}^{2}} \partial_{z}\left(r_{z}^{2} \partial_{z} w\right)=0 .
$$

With, for the fractional integral

$$
y=\int_{0}^{\infty} \mu_{\xi} \varphi \mathrm{d} \xi, \varphi=\varphi(t, z, \xi)
$$

where

$$
\partial_{t} \varphi=-\xi \varphi+\partial_{t} w,
$$

and, for the fractional derivative

$$
\widetilde{y}=\int_{0}^{\infty} \nu(\xi) \partial_{t} \widetilde{\varphi} \mathrm{d} \xi=\int_{0}^{\infty}\left(-\nu(\xi) \xi \widetilde{\varphi}+\nu(\xi) \partial_{t} w\right) \mathrm{d} \xi
$$

with

$$
\partial_{t} \widetilde{\varphi}=-\xi \widetilde{\varphi}+\partial_{t} w \cdot \widetilde{\varphi}=\widetilde{\varphi}(t, z, \xi)
$$

We choose as state variables the energy variables:

$$
\begin{array}{ll}
x_{1}=\partial_{z} w(t, z), & x_{2}=r_{z}^{2} \partial_{t} w(t, z), \\
x_{3}=\varphi(t, z, \xi), & x_{4}=\widetilde{\varphi}(t, z, \xi),
\end{array}
$$

with

- $x_{1}, x_{2} \in L^{2}((a, b) ; \mathbb{R})$,

- $x_{3} \in L^{2}\left((a, b) ; H_{\mu}\right)$, with functional space $H_{\mu}$ defined by $H_{\mu}: \int_{0}^{\infty} \mu(\xi) x_{3}^{2}(\xi,.) \mathrm{d} \xi<\infty$,

- $x_{4} \in L^{2}\left((a, b) ; \widetilde{H}_{\nu}\right)$, with functional space $\widetilde{H}_{\nu}$ defined by $\widetilde{H}_{\nu}: \int_{0}^{\infty} \xi \nu(\xi) x_{4}^{2}(\xi,.) \mathrm{d} \xi<\infty$

The Hamiltonian function $\mathcal{H}\left(x_{1}, x_{2}, x_{3}, x_{4}\right)$ can then be expressed as:

$\mathcal{H}=\frac{1}{2} \int_{a}^{b}\left(r_{z}^{2} x_{1}^{2}+\frac{1}{r_{z}^{2}} x_{2}^{2}+r_{z}^{2} \eta_{z} \int_{0}^{\infty} \mu x_{3}^{2} \mathrm{~d} \xi+r_{z}^{2} \varepsilon_{z} \int_{0}^{\infty} \nu \xi x_{4}^{2} \mathrm{~d} \xi\right)$

In order to define the co-energy variables, we need to define the variational derivative of the Hamiltonian.

\footnotetext{
${ }^{2}$ Indexes $z$ and $\xi$ mean that the parameters depend on the spatial coordinate $z$ or the coordinate $\xi$ respectively.
} 
Definition 4 (Variational derivative of smooth multiscale function). Consider a functional of $x=\left[x_{1}(z), x_{1}(z, \xi)\right]^{T}$ :

$$
\begin{aligned}
\mathcal{H}[x] & =\mathcal{H}\left[x_{1}(z), x_{2}(z, \xi)\right] \\
& =\int_{a}^{b}\left(\mathscr{H}_{1}\left(x_{1}(z)\right)+\int_{0}^{+\infty} \mathscr{H}_{2}\left(x_{2}(z, \xi)\right) \mathrm{d} \xi\right) \mathrm{d} z
\end{aligned}
$$

where $\mathscr{H}_{1}$ and $\mathscr{H}_{2}$ are smooth functions. The multiscale variational derivative of the functional $\mathcal{H}$, denoted by $\frac{\delta \mathcal{H}}{\delta x}$ or $\delta_{x} \mathcal{H}$ is defined as:

$$
\frac{\delta \mathcal{H}}{\delta x}=\left(\begin{array}{l}
\frac{\delta \mathcal{H}}{\delta x_{1}} \\
\frac{\delta \mathcal{H}}{\delta x_{2}}
\end{array}\right)
$$

such that:

$$
\begin{aligned}
& \mathcal{H}[x+\epsilon \delta x]=\mathcal{H}[x]+ \\
& \epsilon \int_{a}^{b}\left(\frac{\delta \mathcal{H}}{\delta x_{1}} \delta x_{1}+\int_{0}^{+\infty} \frac{\delta \mathcal{H}}{\delta x_{2}} \delta x_{2} \mathrm{~d} \xi\right) \mathrm{d} z+O\left(\epsilon^{2}\right)
\end{aligned}
$$

for every $\epsilon \in \mathbb{R}$ and vector of smooth real functions $\delta x(z)$ such that $x+\epsilon \delta x$ satisfies the same boundary conditions as $x$.

In the case of the Webster-Lokshin model the co-energy variables are then defined by:

$$
\begin{array}{ll}
e_{1}=\delta_{x_{1}} \mathcal{H}=r_{z}^{2} \partial_{z} w, & e_{2}=\delta_{x_{2}} \mathcal{H}=\partial_{t} w, \\
e_{3}=\delta_{x_{3}} \mathcal{H}=r_{z}^{2} \eta_{z} \mu_{\xi} \varphi, & e_{4}=\delta_{x_{4}} \mathcal{H}=r_{z}^{2} \varepsilon_{z} \nu_{\xi} \xi \widetilde{\varphi} .
\end{array}
$$

Equation (19) is then "formally" equivalent to:

$$
\left(\begin{array}{c}
\dot{x}_{1} \\
\dot{x}_{2} \\
\dot{x}_{3} \\
\dot{x}_{4}
\end{array}\right)=\left(\begin{array}{ll}
\mathcal{A}_{11} & \mathcal{A}_{12} \\
\mathcal{A}_{21} & \mathcal{A}_{22}
\end{array}\right)\left(\begin{array}{c}
r_{z}^{2} \partial_{z} w \\
\partial_{t} w \\
r_{z}^{2} \eta_{z} \mu_{\xi} \varphi \\
r_{z}^{2} \varepsilon_{z} \nu_{\xi} \xi \widetilde{\varphi}
\end{array}\right)
$$

where

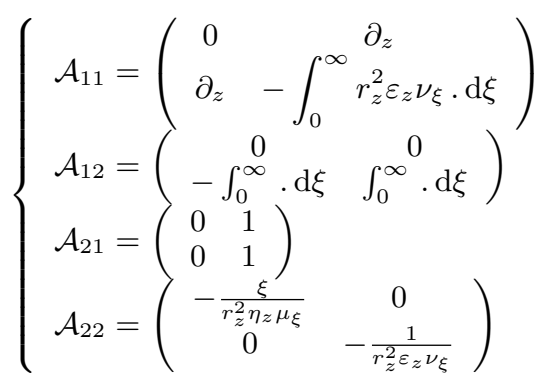

Remark 5. In equation (22), we split the integral of equation (20) into two terms that are not well defined, in fact one must understand the term $\int_{0}^{\infty}\left(\xi \widetilde{\varphi}(t, z, \xi)-\partial_{t} w(t, z)\right) \nu(\xi) \mathrm{d} \xi$ as non separable. Indeed, one could be tempted to split this term into

$$
\left(\int_{0}^{\infty} \xi \nu_{\xi} \widetilde{\varphi}(t, z, \xi) \mathrm{d} \xi\right)-\left(\int_{0}^{\infty} \nu_{\xi} \mathrm{d} \xi\right) \partial_{t} w(t, z)
$$

i.e. of the classical form $y(t, z)=C \widetilde{\varphi}+D \partial_{t} w$; but at least for the fractional operators for which $\nu_{\xi} \propto \xi^{-\alpha}, D=$ $-\int_{0}^{\infty} \nu_{\xi} \mathrm{d} \xi=-\infty$ !

From a geometrical point of view, the dynamical system (22) can be then written in the form:

$$
f=(\mathcal{J}-\mathcal{R}) e
$$

with $e \in \mathcal{E}=H^{1}([a, b], \mathbb{R}) \times H^{1}([a, b], \mathbb{R}) \times L^{2}([a, b], \mathbf{H}) \times$ $L^{2}([a, b], \widetilde{\mathbf{H}}), f \in \mathcal{F}=L^{2}([a, b], \mathbb{R}) \times L^{2}([a, b], \mathbb{R}) \times$ $L^{2}([a, b], \mathbf{H}) \times L^{2}([a, b], \widetilde{\mathbf{H}})$ and operators $\mathcal{J}$ and $\mathcal{R}$ defined as follows:

$$
\mathcal{J}=\left(\begin{array}{cc}
\mathcal{J}_{11} & \mathcal{J}_{12} \\
-\mathcal{J}_{12}^{*} & 0
\end{array}\right)
$$

where

$$
\begin{aligned}
& \mathcal{J}_{11}=\left(\begin{array}{cc}
0 & \partial_{z} \\
\partial_{z} & 0
\end{array}\right), \quad \mathcal{J}_{12}=\left(\begin{array}{ccc}
0 & & 0 \\
-\int_{0}^{\infty} . \mathrm{d} \xi & 0
\end{array}\right) \\
& \mathcal{J}_{12}^{*}=\left(\begin{array}{cc}
0 & -1 \\
0 & 0
\end{array}\right)
\end{aligned}
$$

and

$$
\mathcal{R}=\left(\begin{array}{ll}
\mathcal{R}_{11} & \mathcal{R}_{12} \\
\mathcal{R}_{12}^{*} & \mathcal{R}_{22}
\end{array}\right)
$$

where

$$
\begin{aligned}
& \mathcal{R}_{11}=\left(\begin{array}{cc}
0 & 0 \\
0 & \int_{0}^{\infty} r_{z}^{2} \varepsilon_{z} \nu(\xi) \cdot \mathrm{d} \xi
\end{array}\right) \quad \mathcal{R}_{12}=\left(\begin{array}{cc}
0 & 0 \\
0 & -\int_{0}^{\infty} \cdot \mathrm{d} \xi
\end{array}\right) \\
& \mathcal{R}_{12}^{*}=\left(\begin{array}{cc}
0 & 0 \\
0 & -1
\end{array}\right) \\
& \mathcal{R}_{22}=\left(\begin{array}{cc}
\frac{\xi}{r_{z}^{2} \eta_{z} \mu_{\xi}} & 0 \\
0 & \frac{1}{r_{z}^{2} \varepsilon_{z} \nu_{\xi}}
\end{array}\right)
\end{aligned}
$$

Remark 5 applies to the second line of operator $\mathcal{R}$.

The bond space $\mathcal{B}$ defined as $\mathcal{B}=\mathcal{E} \times \mathcal{F}$ is equipped with the natural power product:

$$
\begin{array}{r}
\left\langle\left(e_{1}, e_{2}, e_{3}, e_{4}\right),\left(f_{1}, f_{2}, f_{3}, f_{4}\right)\right\rangle= \\
\int_{a}^{b}\left(e_{1} f_{1}+e_{2} f_{2}+\int_{0}^{\infty}\left(e_{3} f_{3}+e_{4} f_{4}\right) \mathrm{d} \xi\right) \mathrm{d} z
\end{array}
$$

Lemma 1. $\mathcal{J}$ is formally skew-symmetric and $\mathcal{R}$ is symmetric positive i.e.:

$$
\mathcal{J}=-\mathcal{J}^{*} \text { and } \mathcal{R}=\mathcal{R}^{*}, \mathcal{R} \geq 0
$$

Proof: Let's first consider the skew-symmetry of $\mathcal{J}$ :

$$
\begin{aligned}
& \left\langle e^{\prime}, \mathcal{J} e\right\rangle=\left\langle\left(\begin{array}{cccc}
e_{1}^{\prime} & e_{2}^{\prime} & e_{3}^{\prime} & e_{4}^{\prime}
\end{array}\right),\left(\begin{array}{cc}
\mathcal{J}_{11} & \mathcal{J}_{12} \\
-\mathcal{J}_{12}^{*} & 0
\end{array}\right)\left(\begin{array}{c}
e_{1} \\
e_{2} \\
e_{3} \\
e_{4}
\end{array}\right)\right\rangle \\
& =\int_{a}^{b}\left(e_{1}^{\prime} \partial_{z} e_{2}+e_{2}^{\prime} \partial_{z} e_{1}-e_{2}^{\prime} \int_{0}^{\infty} e_{3} \mathrm{~d} \xi+\int_{0}^{\infty} e_{3}^{\prime} e_{2} \mathrm{~d} \xi\right) \mathrm{d} z \\
& =\int_{a}^{b}\left(-\partial_{z} e_{1}^{\prime} e_{2}-\partial_{z} e_{2}^{\prime} e_{1}+\int_{0}^{\infty} e_{3}^{\prime} e_{2} \mathrm{~d} \xi-e_{2}^{\prime} \int_{0}^{\infty} e_{3} \mathrm{~d} \xi\right) \mathrm{d} z \\
& =\left\langle-\mathcal{J} e^{\prime}, e\right\rangle \text {. }
\end{aligned}
$$

The adjoint operator of $\mathcal{J}$ is equal to $-\mathcal{J}$ and then $\mathcal{J}$ is formally skew-symmetric. In a similar way, one can prove that $\mathcal{R}$ is symmetric i.e.

$$
\begin{aligned}
& \left\langle e^{\prime}, \mathcal{R} e\right\rangle=\left\langle\left(\begin{array}{cccc}
e_{1}^{\prime} & e_{2}^{\prime} & e_{3}^{\prime} & e_{4}^{\prime}
\end{array}\right),\left(\begin{array}{ll}
\mathcal{R}_{11} & \mathcal{R}_{12} \\
\mathcal{R}_{12}^{*} & \mathcal{R}_{22}
\end{array}\right)\left(\begin{array}{c}
e_{1} \\
e_{2} \\
e_{3} \\
e_{4}
\end{array}\right)\right\rangle \\
& =\int_{a}^{b}\left(e_{2}^{\prime} \int_{0}+\infty r_{z}^{2} \varepsilon_{z} \nu e_{2} \mathrm{~d} \xi-e_{2}^{\prime} \int_{0}^{\infty} e_{4} \mathrm{~d} \xi+\int_{0}^{+\infty}\left(e_{3}^{\prime} \frac{\xi e_{3}}{r_{z}^{2} \eta_{z} \mu_{\xi}}\right.\right. \\
& =\left\langle\mathcal{R} e^{\prime}, e\right\rangle . \\
& \left.\left.-e_{4}^{\prime} e_{2}+e_{4}^{\prime} \frac{1}{r_{z}^{2} \varepsilon z \nu_{\xi}} e_{4} \mathrm{~d} \xi\right)\right) d z
\end{aligned}
$$

Moreover, the positivity of $\mathcal{R}$ can be proved as follows:

$$
\begin{aligned}
\langle e, \mathcal{R} e\rangle & =\int_{a}^{b} \int_{0}^{+\infty}\left(e_{2} r_{z}^{2} \varepsilon_{z} \nu_{\xi} e_{2}-e_{2} e_{4}+e_{3} \frac{\xi e_{3}}{r_{z}^{2} \eta_{z} \mu_{\xi}}-e_{4} e_{2}+e_{4} \frac{1}{r_{z}^{2} \varepsilon_{z} \nu_{\xi}} e_{4}\right) \mathrm{d} \xi \\
& =\int_{a}^{b} \int_{0}^{+\infty}\left(\frac{\xi}{r_{z}^{2} \eta_{z} \mu_{\xi}} e_{3}^{2}+\left(r_{z} \sqrt{\varepsilon_{z} \nu} e_{2}-\frac{1}{r_{z} \sqrt{\varepsilon_{z} \nu}} e_{4}\right)^{2} \mathrm{~d} \xi\right) \mathrm{d} z \geq 0
\end{aligned}
$$

Of course, $\mathcal{R}$ is not even positive definite, thus never coercive.

System (23) can be written in the form of an extended system with closure equation related to the dissipation by 
using the extended operator $\mathcal{J}_{e}$ with $\left(\begin{array}{c}f \\ f_{s}\end{array}\right)=\mathcal{J}_{e}\left(\begin{array}{c}e \\ e_{s}\end{array}\right)$ with $e_{s}=S f_{s}$, where:

$\mathcal{J}_{e}=\left(\begin{array}{ccccccc}0 & \partial_{z} & 0 & 0 & 0 & 0 & 0 \\ \partial_{z} & 0 & -\int_{0}^{\infty} . \mathrm{d} \xi & 0 & -\int_{0}^{\infty} . \mathrm{d} \xi & 0 & 0 \\ 0 & 1 & 0 & 0 & 0 & -1 & 0 \\ 0 & 0 & 0 & 0 & 0 & 0 & 1 \\ 0 & 1 & 0 & 0 & 0 & 0 & 0 \\ 0 & 0 & 1 & 0 & 0 & 0 & 0 \\ 0 & 0 & 0 & -1 & 0 & 0 & 0\end{array}\right)$,

and

$$
S=\left(\begin{array}{ccc}
r_{z}^{2} \varepsilon_{z} \nu_{\xi} & 0 & 1 \\
0 & \frac{\xi}{r_{z}^{2} \eta_{z} \mu_{\xi}} & 0 \\
1 & 0 & \frac{1}{r_{z}^{2} \varepsilon_{z} \nu_{\xi}}
\end{array}\right)
$$

with $e_{s} \in \mathcal{E}_{s}=H^{1}([a, b], \mathbb{R}) \times L^{2}([a, b], \mathbf{H}) \times L^{2}([a, b], \widetilde{\mathbf{H}})$, $f_{s} \in \mathcal{F}_{s}=L^{2}([a, b], \mathbb{R}) \times L^{2}([a, b], \mathbf{H}) \times L^{2}([a, b], \widetilde{\mathbf{H}})$.

One can check that $\mathcal{J}_{e}$ is formally skew-symmetric and $S$ symmetric positive i.e. :

$$
\mathcal{J}_{e}^{*}=-\mathcal{J}_{e} \text { and } S=S^{*} \geq 0
$$

We now consider systems with non zero boundary flow. One can naturally extend the effort and the flow spaces to include the boundary, by defining:

$$
\begin{aligned}
& \mathscr{E}=\mathcal{E}_{e} \times \mathbb{R}^{2}=\mathcal{E} \times \mathcal{E}_{s} \times \mathbb{R}^{2} \\
& \mathscr{F}=\mathcal{F}_{e} \times \mathbb{R}^{2}=\mathcal{F} \times \mathcal{F}_{s} \times \mathbb{R}^{2}
\end{aligned}
$$

We define a symmetric pairing from the power product by:

$$
\begin{aligned}
\left\langle\left(e_{e}, e_{\partial}, f_{e}, f_{\partial}\right),\left(\tilde{e}_{e}, \tilde{e}_{\partial}, \tilde{f}_{e}, \tilde{f}_{\partial}\right)\right\rangle_{+} & =\left\langle\left(e_{e}, \tilde{f}_{e}\right)\right\rangle+\left\langle\left(\tilde{e}_{e}, f_{e}\right)\right\rangle \\
& -\left\langle\left(\tilde{e}_{\partial}, f_{\partial}\right)\right\rangle-\left\langle\left(e_{\partial}, \tilde{f}_{\partial}\right)\right\rangle
\end{aligned}
$$

with $\left(e_{e}, e_{\partial}, f_{e}, f_{\partial}\right)$ and $\left(\tilde{e}_{e}, \tilde{e}_{\partial}, \tilde{f}_{e}, \tilde{f}_{\partial}\right) \in \mathscr{B}=\mathscr{E} \times \mathscr{F}$.

In order to define a Dirac structure we need to define appropriate boundary port variables with respect to the considered differential operator and symmetric pairing. In [Villegas et al.(2006)] a parametrization of boundary port variables is given in the case of non full rank linear differential operators. This parametrization can be adapted to our case study as follows:

Definition 5. Considering the following parametrization of $\mathcal{J}_{e}$ :

$$
\mathcal{J}_{e}=P_{1} \partial_{z}+P_{0}
$$

with

$$
P_{1}=\left(\begin{array}{cc}
\Sigma_{2} & 0_{2,5} \\
0_{5,2} & 0_{5,5}
\end{array}\right)
$$

$$
P_{0}=\left(\begin{array}{ccccccc}
0 & 0 & 0 & 0 & 0 & 0 & 0 \\
0 & 0 & -\int_{0}^{\infty} . \mathrm{d} \xi & 0 & -\int_{0}^{\infty} . \mathrm{d} \xi & 0 & 0 \\
0 & 1 & 0 & 0 & 0 & -1 & 0 \\
0 & 0 & 0 & 0 & 0 & 0 & 1 \\
0 & 1 & 0 & 0 & 0 & 0 & 0 \\
0 & 0 & 1 & 0 & 0 & 0 & 0 \\
0 & 0 & 0 & -1 & 0 & 0 & 0
\end{array}\right)
$$

with $\Sigma_{2}=\left(\begin{array}{ll}0 & 1 \\ 1 & 0\end{array}\right)$, the boundary port variables associated with the differential operator $\mathcal{J}_{e}$ are the vectors $f_{\partial}, e_{\partial} \in \mathbb{R}^{2}$ given by:

$$
\begin{aligned}
&\left(\begin{array}{l}
f_{\partial} \\
e_{\partial}
\end{array}\right)=\frac{1}{\sqrt{2}}\left(\begin{array}{cc}
\Sigma_{2} & -\Sigma_{2} \\
I_{2} & I_{2}
\end{array}\right) \\
&\left(\begin{array}{cccc}
I_{2} & 0_{2,5} & 0_{2,2} & 0_{2,5} \\
0_{2,2} & 0_{2,5} & I_{2} & 0_{2,5}
\end{array}\right)\left(\begin{array}{l}
e_{e}(b) \\
e_{e}(a)
\end{array}\right)
\end{aligned}
$$

The definition of the boundary port variables gives rise to the definition of the associated Dirac structure.

Theorem 2. The subspace $\mathcal{D}_{\mathcal{J}_{e}}$ of $\mathscr{B}$ defined as:

$$
\begin{aligned}
\mathcal{D}_{\mathcal{J}_{e}} & =\left\{\left(\begin{array}{c}
f_{e} \\
f_{\partial} \\
e_{e} \\
e_{\partial}
\end{array}\right) \mid f_{e}=\mathcal{J} e_{e}\right. \text { and } \\
& \left.\left(\begin{array}{c}
f_{\partial} \\
e_{\partial}
\end{array}\right)=\frac{1}{\sqrt{2}}\left(\begin{array}{cccc}
\Sigma_{2} & 0_{2,5} & -\Sigma_{2} & 0_{2,5} \\
I_{2} & 0_{2,5} & I_{2} & 0_{2,5}
\end{array}\right)\left(\begin{array}{c}
e_{e}(b) \\
e_{e}(a)
\end{array}\right)\right\}
\end{aligned}
$$

is a Dirac structure with respect to the product (25).

Proof: We used the parametrization proposed in [Villegas et al.(2006)] to define some boundary port variables such that the symmetric pairing (25) is non degenerate and $\mathcal{D}_{\mathcal{J}_{e}}$ is a Dirac structure, i.e.

$$
\mathcal{D}_{\mathcal{J}_{e}}=\mathcal{D}_{\mathcal{J}_{e}}^{\perp}
$$

Such parametrization arises from the integration by part of the skew differential operator, the projection of the image space and the definition of the inner product.

\section{Perspectives}

In order to develop this research theme in the near future, the following tracks could be investigated, either on the physical side, the theoretical side, or even the numerical side:

1) work out the derivation of the physical model itself: see e.g. [Bruneau et al.(1989)], [Polak(1991)] and also reference works on thermodynamics in a port Hamiltonian framework, to understand if a better formulation could be possible: in this case, a better formulation would mean closer to elementary physical principles.

2) try to develop an asymptotic analysis approach to formulate the model of [Polak(1991)], and see to what extent some port-Hamiltonian setting can be compatible with this asymptotic analysis (in which case the small parameter would be the diffusive coefficient $\eta$ or $\varepsilon$ in the constant case)?

3) possibly examine other examples of physical models with fractional derivatives, to see to what extent they can be recast in the framework developped in this paper: coupling between a conservative system and a diffusive system. One model of interest could be the Biot and Johnson-Koplik-Dashen (JKD) models for waves in porous media, see e.g. [Blanc et al.(2013)] and references therein.

4) investigate the numerics: a first approach has been proposed in [Haddar et al.(2010)] and fully detailed in [Haddar et al.(2008), chap. 3], but another way of addressing the numerics could be first to apply a 
Finite Element Method (FEM) respecting the geometrical structure of the Hamiltonian formulation, as in [Moulla et al. (2012)], second to use the Finite Difference Method (FDM) adapted to fractional dynamics, such as in [Deü et al.(2010)]. Moreover, for the treatment of the time-domain part, advantage should be taken from so-called symplectic methods for Hamiltonian systems, first when no dissipation is present, see e.g. [Leimkuhler et al.(2004)], and recently used with dissipation in [Hu et al. (2013)].

5) analyse the asymptotic stability, see e.g. [Villegas et al.(2006)] for the principles, but a lack of compactness of the resolvent operator prevents the use of standard techniques; hence, for fractional systems, special care must be taken, see [Matignon et al.(2005)] for a fractional ODE on a single mode, or even more so for the fractional PDE, as presented first in [Matignon(2006)], and finally in [Matignon et al.(2013a)].

6) look carefully at the domains of the operators, in particular with the fractional derivative, where two parts cannot be treated separately, as seen in the functional analytic framework developped in [Haddar et al.(2008), chap. 2]: this reminds of the so-called non separable $C \& D$ observation operators developped in some operator theoretic frameworks. This theoretical point, put forward in Remark 5, must be tackled seriously.

\section{CONCLUSION}

In this paper, we propose a port-Hamiltonian formulation of systems arising from the coupling of a wave equation with a diffusion equation related to acoustic phenomena. The considered diffusion equation contains a fractional derivative in time and physical coefficients variable in space. First we consider the finite dimensional approximation of the integral and fractional derivatives. It is based on a diffusive representation of integral and fractional derivatives. In a second instance, we consider the Webster-Lokshin equation that is made up by the coupling of the wave equation and the aforementionned diffusion term. From the definition of the energy variables, Hamiltonian function and power conjugate flow and effort vectors, we propose the definition of some appropriate boundary port variables in order to define a Dirac structure. This Dirac structure allows to connect the internal energetic behavior of the system with the power flow at the boundary. This first work on the geometrical formulation of such system will open to the use of functional analysis tools that have been previously derived in the context of differential systems with dissipation in [Zwart et al.(2011)]. Nevertheless a particular care will have to be taken on the characterization of functional spaces, particularly in the case of the diffusion function for which the domain is not separable, as already taken care of in [Haddar et al.(2008)].

\section{REFERENCES}

[Baaiu et al.(2009)] A. BaAiu, F. Couenne, D. Eberard, C. Jallut, Y. Le Gorrec, L. Lefevre, and M. Tayakout- Fayolle. Port- based modelling of mass transfer phenomena. Mathematical and Computer Modelling of Dynamical Systems, 15(3):233254, 2009.

[Blanc et al.(2013)] E. Blanc, G. ChiAvass A, AND B. Lombard, BiotJKD model: simulation of $1 D$ transient poroelastic waves with fractional derivatives, J. Comput. Phys., vol. 237 (2013), pp. 1-20.

[Bruneau et al.(1989)] M. Bruneau, Ph. Herzog, J. Kergomard, AND J.-D. POLACK, General formulation of the dispersion equation in bounded visco-thermal fluid, and application to some simple geometries, Wave Motion, vol. 11 (1989), pp. 441-451.

[Deü et al.(2010)] J.-F. DEÜ AND D. MATIGNON, Simulation of fractionally damped mechanical systems by means of a Newmark-diffusive scheme, Computers and Mathematics with Applications, vol. 59 (2010), pp. 1745-1753.

[Duindam et al.(2009)] Duindam, V., Macchelli, A., Stramigioli, S., and Beruyninckx, H. (eds.) (2009). Modeling and Control of Complex Physical Systems. The Port-Hamiltonian approach. Spinger Verlag.

[Haddar et al.(2010)] H. HADDAR, J. R. LI, AND D. MATIGNON, Efficient solution of a wave equation with fractional-order dissipative terms, Journal of Computational and Applied Mathematics, vol. 234 (2010), pp. 2003-2010.

[Haddar et al.(2004)] H. HADDAR AND D. MATIGNON, Well-posedness of non-linear conservative systems when coupled with diffusive systems, in IFAC symposium on Nonlinear Control Systems (NOLCOS), vol. 1, Stuttgart, Germany, sept 2004.

[Haddar et al.(2008)] - Theoretical and numerical analysis of the Webster-Lokshin model, Research Rep. RR-6558, Institut National de la Recherche en Informatique et Automatique (INRIA), 2008. http ://hal.inria.fr/inria-00288254_v2/

[Hélie et al.(2006)] T. HÉLIE AND D. MATIGNON, Diffusive representations for the analysis and simulation of flared acoustic pipes with visco-thermal losses, Math. Models Meth. Appl. Sci., 16 (2006), pp. 503-536.

[Hu et al. (2013)] W. Hu, Z. DEng, S. HAN AND W. ZHANG, Generalized multi-symplectic integrators for a class of Hamiltonian nonlinear wave PDEs. J. Comput. Phys., vol. 235 (2013), pp. 394-406.

[Jacob et al.(2012)] B. JACOB AND H.J. ZwART, Linear Port Hamiltonian Systems on Infinite-dimensional Spaces, Volume 223 of Operator Theory: Advances and Applications. Birkhauser, Springer Basel, 2012.

[Kergomard et al.(2013)] J. Kergomard, D. LAFARGE AND J. GILBERT Transients in porous media: exact and modelledtime-domain Green's functions. Acta Acustica united with Acustica To appear, 2013.

[Le Gorrec et al.(2005)] Y. Le Gorrec, H. Zwart AND B. MaschKe. Dirac structures and Boundary Control Systems associated with SkewSymmetric Differential Operators. SIAM Journal on Control and Optimization, Vol: 44 Issue 5 (2005), pp. 1864-1892.

[Le Gorrec et al.(2006)] Y. Le Gorrec, J. A. Villegas, H. ZwArt, AND B. MAschKe, Dissipative Boundary Control Systems with Application to Distributed Parameters Reactors Joint CCA, ISIC and CACSD 2006, October 4-6, 2006, Munich, Germany.

[Le Gorrec et al.(2012)] Y. Le Gorrec, And D. Matignon, Diffusive systems coupled to an oscillator: a Hamiltonian formulation. In IFAC conf. on Lagrangian and Hamiltonian Methods and Nonlinear Control (LHMNLC'12), August 29-31, 2012, Bertinoro, Italy. (invited session).

[Leimkuhler et al.(2004)] B.LEIMKUHLER AND S. REICH, Simulating Hamiltonian dynamics, Cambridge University Press, 2004,

[Matignon(2006)] D. MAtignon, Asymptotic stability of the WebsterLokshin model. In Mathematical Theory of Networks and Systems (MTNS), 2006, Kyoto, Japan. (invited session).

[Matignon(2009a)] D. MATIGNON, An introduction to fractional calculus, vol. 1 of Scaling, Fractals and Wavelets of Digital Signal and Image Processing Series, ISTE-Wiley, (2009), pp. 237-278.

[Matignon(2009b)] D. MatignON, Diffusive representations for fractional Laplacian: systems theory framework and numerical issues, Physica Scripta, vol. T136 (2009), p. 014009.

[Matignon et al.(2005)] D. MATIGNON AND C. PRIEUR, Asymptotic stability of linear conservative systems when coupled with diffusive systems, ESAIM Control Optim. Calc. Var., 11 (2005), pp. 487-507.

[Matignon et al.(2013a)] D. MATIGnon And C. Prieur, Asymptotic stability of Webster-Lokshin model, (2013), submitted.

[Matignon et al.(2013b] D. Matignon And H. ZwART, Standard diffusive systems as well-posed linear systems. International Journal of Control, (2013). To appear.

[Moulla et al. (2012)] R. Moulla, L. Lefèvre, AND B. MaschKe, Pseudo-spectral methods for the spatial symplectic reduction of open 
systems of conservation laws. J. Comput. Phys., vol. 231 (2012), pp. 1272-1292.

[Polak(1991)] POLACK, J.D, (1991). Time domain solution of Kirchhoff's equation for sound propagation in viscothermal gases: a diffusion process. J. Acoustique, 4 (1991), pp. 47-67.

[van der Schaft and Maschke(2004)] van der Schaft, A. and Maschke, B. (2004). Advanced Dynamics and Control of Structures, volume 444 of CISM International Centre for Mechanical Sciences, chapter PortHamiltonian systems: network modeling and control of nonlinear physical systems. Springer.

[Villegas et al.(2006)] J. VillegAs, Y. LE GorREC, H. ZwART, AND B. MASCHKE, Boundary control for a class of dissipative differential operators including diffusion systems. In Mathematical Theory of Networks and Systems (MTNS), July 24-28, 2006, Kyoto, Japan. (invited session)

[Villegas et al.(2009)] J. A. Villegas, H. ZWART, Y. LE GorReC, AND B. MASCHKE. Exponential Stability of a Class of Boundary Control Systems. IEEE Transactions on Automatic Control- Vol. 54(1), Pages : 142-147, January 2009.

[van der Schaft et al.(2002)] A.J. VAN DER SCHAFT AND B.M. MASCHKE, Hamiltonian formulation of distributed parameter systems with boundary energy flow. In J. of Geometry and Physics, Vol: 42, Pages: 166-174, 2002.

[Zwart et al.(2010)] H. ZwART, Y. Le Gorrec, B. MaschKe AND J. VILLEGAS. Well-posedness and regularity of hyperbolic boundary control systems on a one-dimensional spatial domain. ESAIM Control, Optimisation and Calculus of Variations, Vol. 16, Pages : 1077-1093, 2010.

[Zwart et al.(2011)] H. Zwart, Y. LE Gorrec AND B. MASChKe, Linking Hyperbolic and Parabolic PDEs Proceedings of 50th IEEE Conference on Decision and Control and European Control Conference, CDC-ECC 2011, Pages: 4921-4924, 12-15 December 2011, Orlando, FL, USA. 